\title{
Deep MS/MS-Aided Structural-similarity Scoring for Unknown Metabolites Identification
}

\author{
Hongchao Ji, Yamei Xu, Hongmei Lu*, Zhimin Zhang*. \\ College of Chemistry and Chemical Engineering, Central South University, Changsha 410083, PR China.
}

Corresponding Author E-mail address: hongmeilu@csu.edu.cn; zhangzhimin@ @ csu.edu.cn

\section{The detail of MetFrag comparison of cross validation test.}

MetFrag. When retrieving candidates from metabolite database, since $\mathrm{R}$ version of MetFrag does not support custom database, we used $\mathrm{R}$ to write config file and call command line version of MetFrag (2.4.2, http://c-ruttkies.github.io/MetFrag/projects/metfragcl). The source codes for processing with MetFrag are available at https://github.com/hcji/DeepMASS/tree/master/support. When retrieving candidates from in PubChem database, we used $\mathrm{R}$ version MetFrag with 2.4.2 version (https://github.com/c-ruttkies/MetFragR). We set the database for retrieving candidates as PubChem. When searching candidates, the true formula and exact mass were given. The rest parameters were kept as default. MetFrag cannot process all spectra, 16 spectra were failed because of crashing or omitting the correct answer from the output list. When calculating the rate, those spectra were not count. When MetFrag gave the same score to more than one candidate, which was a very common condition, we tied them for the same highest rank.

CFM-ID. When retrieving candidate, we retrieved candidates with formula both from metabolite database and PubChem database and wrote them to config file. Then we wrote python scripts to call command line version of CFM-ID 2.4 (https://sourceforge.net/projects/cfm-id/). The source codes for processing with CFM-ID software are available at https://github.com/hcji/CFM_ID_pywrapper. For the parameters, the mass tolerance was set as $0.01 \mathrm{Da}$, possibility threshold was set as 0.001 and the score type was set as Jaccard. 10 spectra were failed when processing with CFM-ID and candidates from PubChem because of background shutdown unexpectedly. Maybe it was caused by inputting too many candidates, for example, there are 16,709 candidates for $\mathrm{C} 05643$ and 18,723 candidates for $\mathrm{C} 1598$. When calculating the rate, those spectra were not count. If CFM-ID gave the same score to more than one candidate, we tied them for the same highest rank.

\section{Brief introduction of the male infertility study.}

Semen samples of fertile and infertile males were collected from Xiangya Hospital of Central South University. After thawed at $4{ }^{\circ} \mathrm{C}$ in refrigerator for 30 minutes, seminal plasma $(100 \mu \mathrm{L})$ 
was ready for use. For solvent extraction, seminal plasma was placed in a $1.5 \mathrm{~mL}$ centrifuge tube, then added with $400 \mu \mathrm{L}$ mixed solvent which contained chloroform, methanol and water $(\mathrm{V} / \mathrm{V} / \mathrm{V}=2: 1: 1)$. The mixtures were vortexed for $15 \mathrm{~min}$, and then centrifuged at $16000 \mathrm{rpm}$ for 5 min. After centrifugation, $180 \mathrm{uL}$ of the upper layer was transferred to a clean centrifuge tube, dried with $\mathrm{N} 2$ gas to $100 \mu \mathrm{L}$. $150 \mu \mathrm{L}$ lower layer solution was transferred to another clean centrifuge tube, and dried with N2 gas. Dried lower phase was reconstituted with $100 \mathrm{uL}$ methanol and then combined with $100 \mu \mathrm{L}$ upper phase as the final solution for LC-MS detection.

LC-MS analysis was performed on an LC-30AD UHPLC system (Shimadzu, Japan) coupling with Ion Trap-Time of Flight (IT-TOF) mass spectrometer (Shimadzu, Japan). For chromatographic separation of an ACQUITY UPLC HSS C18 $(2.1 \times 100 \mathrm{~mm}, 1.8 \mu \mathrm{m}$, Waters Corporation, USA) column was used. The mass spectrometer was operated in the full scan and automatic multiple stage fragmentation scan modes over an $\mathrm{m} / \mathrm{z}$ range of $10-1000$ in positive ion modes. Capillary voltage was set at $4,500 \mathrm{~V}$. Nebulizing gas (N2) was applied at a $1.5 \mathrm{~L} / \mathrm{min}$ flow rate; drying gas (N2) was applied at a $100 \mathrm{KPa}$ pressure.

Finally, biomarkers were selected after data processed and statistical analysis. The selected biomarkers were identified by comparing spectra with standards or query HMDB spectra database. The same sample were also measured by a Bruker LC-ESI-QTOF-MS instrument with MRM mode under the same setting, for collecting the MS/MS spectra from another type of instruments.

\section{The detail of comparison of external data test.}

MetFrag. We used the command line version of MetFrag, and used the metabolite database for retrieving candidates. Other parameters were kept as default.

CFM-ID. For We used the web server at http $/ /$ cfmid.wishartlab.com/identify. We retrieved the candidates from the metabolite database based on the molecular formula manually and input them as candidates used by CFM-ID. The mass tolerance was set as $0.01 \mathrm{Da}$. Each spectrum was input as 'low energy', 'medium energy' and 'high energy', respectively and the best results were used.

MAGMa. We used the web server at http://www.emetabolomics.org/magma . The spectra were transformed to MGF format for input. We retrieved the candidates from the metabolite database based on the molecular formula manually. Then we uploaded the candidates by SMILES. The mass tolerance was also set as $0.01 \mathrm{Da}$. Other parameters were kept as default.

MS-Finder. We used the 3.12 version MS-Finder, which was download from http://prime.psc.riken.jp/Metabolomics_Software/MS-FINDER. The spectra were input by creating queries. Only the metabolite database is used, and the mass tolerance was also set as 0.01 Da. The relative abundance cut off of MS/MS was set as $1 \%$. Other parameters were kept as default.

\section{Reason for the selection of CFM-ID for generating the theoretical MS/MS spectra}


MetFrag, CFM-ID, MAGMa, MIDAS and MS-Finder are commonly used to generate in silicon MS/MS spectra for unknown metabolites identification. MetFrag and MS-Finder generate the superset of potential fragments and use the reverse matching method for identification. It means that the theoretical spectra generated by MetFrag and MS-Finder are not suitable for this study. From Table 2 of MS-Finder paper, the computation cost of MIDAS is the highest among the five methods. MAGMA is a web-application, and its performance depends on network, server conditions and varying over time. Therefore, CFM-ID is chosen to generate theoretical mass spectra in this study. 